\title{
Infecção de sementes de trigo com Bipolaris sorokiniana pela técnica de restrição hídrica
}

\author{
Cândida R.J. de Farias', Emerson M. Del Ponte², Carla L. Corrêa ${ }^{3}$, Ana Paula Afonso ${ }^{3}$ \& Carlos R. \\ Pierobom $^{3}$
}

${ }^{1}$ Universidade Federal de Pelotas - Faculdade de Agronomia “Eliseu Maciel”, 96010-900, Pelotas, RS, Brasil; ${ }^{2}$ Departamento de Fitossanidade, Universidade Federal do Rio Grande do Sul, Porto Alegre, RS, Brasil; ${ }^{3}$ Departamento de Fitossanidade, Faculdade de Agronomia, Universidade Federal de Pelotas, Pelotas, RS, Brasil

Autor para correspondência: Cândida R.J. de Farias, e-mail: candidajacobsen@bol.com.br

\section{RESUMO}

A inoculação de fungos fitopatogênicos em sementes pela técnica da restrição hídrica é relativamente recente. Este trabalho objetivou avaliar o efeito de potenciais osmóticos (-0.4, -0,6 e -0.8) do substrato BDA, modificado por adição de sacarose, em interação com o tempo de exposição (46, 72 e 96 h), na infeção de sementes de trigo por Bipolaris sorokiniana. Foram analisadas a eficiência da infecção e a inibição da germinação durante e após a aplicação dos tratamentos. De acordo com os resultados, períodos de tempo superiores a 48 horas de exposição à colônia fúngica não foram inibitórios a germinação completa das sementes, a qual incrementou linearmente com o aumento do tempo de contato com o inóculo fúngico. A eficiência de infecção não diferiu para os potenciais osmóticos e tempo de exposição, variando de 0,53 a 0,92 . No entanto, a capacidade germinativa das sementes após os tratamentos foi significativamente reduzida após o período de 48 horas de exposição das sementes ao substrato modificado contendo a cultura fúngica.

Palavras-chave: potencial osmótico, sanidade de sementes, inoculação artificial.

\section{ABSTRACT \\ Infection of wheat seeds by Bipolaris sorokoniana using a water restriction technique}

Seed inoculation with pathogenic fungi using the water restriction technique is relatively recent. This study aimed to verify the effect of osmotic potentials $(-0.4,-0.6$ and $-0.8 \mathrm{MPa})$ of PDA substrate modified by the amendment of sucrose in interaction with the period of exposure $(48,72$ and $96 \mathrm{~h})$ of wheat seeds to colonies of Bipolaris sorokiniana. Infection efficiency and inhibition of seed germination during and after treatment were the variables analyzed. The results of this study show that an exposure period longer than 48 hours to the fungal colonies did not inhibit full germination of the seeds. The germination rate increased linearly as the exposure period to the fungal colony was increased. Infection efficiency was not affected by osmotic potential and exposure time, varying from 0.53 to 0.92 . However, seed germination was significantly reduced after 48 hours of exposure to the fungal colonies.

Key words: osmotic potential, seed health, artificial inoculation.

A disponibilidade de sementes infectadas é essencial para a condução de diversos estudos no âmbito da patologia de sementes como epidemiologia de doenças transmitidas por sementes, metodologia de detecção de patógenos, teste de eficiência de tratamento de sementes, dentre outros (Machado, 1988). No entanto, lotes contaminados nem sempre estão disponíveis prontamente ou contém o tipo de patógeno e os níveis desejados de infecção para uma experimentação.Ainfecçãode plantas adultas paraa produção de sementes contaminadas sob condições controladas, além de laboriosa e demorada, tem sua eficiência questionada em função dos vários fatores que afetam o processo de infecção em situação próxima à natural (Neergard, 1979; Machado et al., 2001). Alternativamente, a inoculação artificial da semente destaca-se como alternativa viável para garantir o desenvolvimento potencial da doença a partir de sementes contaminadas. Contudo, dependendo da metodologia, a contaminação pode ser apenas superficial à semente, principalmente se conduzida com métodos tradicionais como a embebição das sementes em suspensão de conídios ou pelo contato direto das sementes com a colônia fúngica em meio de cultura (Machado et al., 2004).

A modificação do potencial osmótico do substrato do meio de cultura para controlar a germinação de sementes em testes de sanidade, técnica denominada de restrição hídrica (Coutinho et al., 2001; Machado \& Langerack, 2002; Machado et al., 2008), tem recebido bastante atenção nos últimos anos, devido à suas vantagens em comparação aos métodos convencionais de detecção (Farias et al., 2003; Machado et al., 2004, 2007). A possibilidade de se controlar a germinação das sementes levou a proposição de uma metodologia de inoculação que permite promover diferentes níveis de infecção de sementes quando estas são postas em contato com a colônia fúngica em meios de 
cultura ajustados em seu potencial osmótico (Costa et al., 2003; Machado et al., 2004, Araujo et al., 2006).

$\mathrm{Na}$ otimização da técnica de inoculação artificial sob restrição hídrica do sustrato torna-se importante a determinação do período de tempo de exposição das sementes, de forma que inibia a germinação, característica desejável, mas que principalmente garanta o nível de infecção almejado e sem afetar negativamente o poder germinativo da semente (Machado et al., 2001, 2004).

O fungo Bipolaris sorokiniana (Sacc.) Shoemaker (teleomorfo Cochliobolus sativus) destaca-se dentre os mais importantes patógenos de sementes de trigo e cevada no sul do Brasil (Reis et al., 1988; Toledo et al., 2002). Sua introdução em áreas novas ou sob rotação de culturas é preocupante devido à sua alta eficiência de transmissão para diferentes órgãos radiculares e aéreos, que varia de 38 a 88\% (Reis \& Forcelini, 1993). Estudos anteriores com modificações do substrato para cultivo e detecção de fungos demonstraram a não inibição do desenvolvimento de $B$. sorokiniana em substrato com restrição hídrica (Farias et al., 2003), e um estímulo ao seu crescimento em meio de cultura modificado osmoticamente com adição de sacarose (Farias et al., 2004). Neste trabalho objetivouse avaliar a infecção artificial de sementes de trigo por $B$. sorokiniana por meio da metodologia de restrição hídrica, considerando-se o efeito de níveis de potenciais osmóticos do substrato modificado com sacarose em interação com períodos variados de incubação de sementes sobre colônias do fungo.

Uma cultura pura de B. sorokiniana, isolada de semente de trigo severamente contaminada, e conservada na coleção do Departamento de Fitossanidade, da Faculdade de Agronomia da Universidade Federal de Pelotas (código BsT01), foi utilizada como inóculo. A sua multiplicação foi realizada por transferências de fragmentos de micélio a partir de um tubo de ensaio para placas de Petri de plástico, de $9 \mathrm{~cm}$ de diâmetro, contendo meio BDA(Batata-DextroseÁgar), incubadas por 10 dias a $25^{\circ} \mathrm{C}$ e fotoperíodo de 12 horas de luz. Lotes de sementes cedidos pela Embrapa Clima Temperado, Pelotas RS, da safra 2003, foram analisados previamente para a escolha de um lote em que não se observou a presença de $B$. sorokiniana nas sementes, para a condução do experimento.

A sacarose, substância de baixo custo e amplo uso em laboratório, foi escolhida para promover a restrição hídrica no meio BDA, cujos procedimentos de preparo e quantidade de sacarose a adicionar seguiram a metodologia descrita em trabalhos anteriores (Farias et al., 2003, 2004). Discos de micélio $(0,8 \mathrm{~mm})$ de $B$. sorokiniana foram colocados no centro de placas de petri contendo BDA, incubadas a seguir por um período de cinco dias. Após o período de crescimento, 200 sementes foram depositadas em camada única sobre a cultura crescida em cada placa, retornando em seguida para a câmara de crescimento. Os fatores de influência testados foram o potencial osmótico do substrato, em três níveis: $-0,4 ;-0,6$ e $-0,8 \mathrm{MPa}$ e tempo de exposição das sementes à colônica fúngica, também em três níveis: 48, 72 e $96 \mathrm{~h}$. O delineamento experimental foi o inteiramente casualizado com os tratamentos distribuídos em esquema fatorial $3 \times 3$ com quatro repetições, compostas por 200 sementes cada.

Após o término de cada período de exposição nos tratamentos as sementes não germinadas foram coletadas, desinfestadas com hipoclorito de sódio (1\% por 2 minutos) e lavadas por três vezes com água destilada, antes de serem submetidas ao teste de sanidade em substrato de papel de filtro. Esse foi conduzido sem modificações para a inibição de germinação e procedimento padrão de incubação por sete dias a $25 \pm 2^{\circ} \mathrm{C}$ e 12 horas de fotoperíodo.

Foram utilizadas quatro repetições de 200 sementes, sendo cada repetição subdividida em oito amostras de 25 sementes cada, obtendo-se dessa forma a média. Em seguida, as sementes foram individualmente examinadas com auxílio de microscópio estereoscópico para a observação da presença de crescimento de B. sorokiniana sobre a semente, proveniente de infecção interna. A eficiência de infecção representa a proporção de sementes infectadas em relação ao total de sementes inoculadas. O percentual de germinação das sementes foi observado na ocasião da leitura da eficiência da infecção.

Para a análise estatística, os dados de germinação e de eficiência de infecção foram transformados em log $\mathrm{x}+1$ quando verificada ausência de normalidade na distribuição dos dados. Os mesmos foram submetidos à análise de regressão por superfície de resposta. O modelo foi validado pela análise de variância verificando-se o coeficiente de determinação e teste $\mathrm{F}$. O nível de significância adotado foi de $95 \%$ de confiança. As análises foram conduzidas nos programas Statistica 7.0

A inibição completa da germinação das sementes, durante o tratamento de inoculação, foi observada apenas nos tratamentos com exposição da sementes no tempo mínimo de 48 horas. Foi observada uma tendência de aumento no percentual de germinação com o aumento do tempo de exposição das sementes ao inóculo. No entanto, baixa germinação $(6,3 \%)$ foi observada no maior potencial osmótico, mesmo no período mais longo avaliado, 96 horas. A resposta da germinação aos fatores testados foi modelada por uma função quadrática com fatores em interação $\left(\mathrm{R}^{2}=0.98\right.$ e $\mathrm{R}^{2}$ ajustado=0.95; $\left.\mathrm{P}<0.01\right)$ (Figura 1$)$.

De acordo com Bradford (1986), a emergência da radícula ocorre quando o conteúdo de água da semente atinge um platô em função de um potencial hídrico de equilíbrio específico entre as sementes e o meio externo. A restrição hídrica, portanto, modifica as condições originais do meio, causando o atraso na germinação das sementes no tratamento com maior potencial osmótico (Coutinho et al., 2001).

Todos os tratamentos promoveram a infecção das sementes a partir de $48 \mathrm{~h}$ de exposição das sementes à colônia do fungo. A eficiência média de infecção variou de 0,53 a 0,92 , porém sem significância na análise de 
regressão para quaisquer dos fatores avaliados $(\mathrm{P}>0,05)$. No entanto, os valores médios absolutos mais altos de infecção foram observados no maior período de exposição (96h) e, conjuntamente, nos tratamentos com potencial osmótico do substrato em -0,6 MPa (Figura 2).

A eficiência da infecção de fungos em sementes de feijoeiro utilizando a metodologia de restrição hídrica foi demonstrada por Costa et al. (2003) inoculando Fusarium oxysporum f.sp. phaseoli, quando foram avaliados os tempos de 36 a 144h de exposição das sementes ao inóculo. Os autores observaram que a incidência do fungo foi mais alta com o aumento do tempo de exposição das sementes: $24,3 \%$ e $70 \%$ para o menor e o maior tempo de exposição da semente ao fungo, respectivamente.

$z=1.7907-0.0495^{*} x-1.0352^{*} y+0.0005^{*} x^{*} x-0.0077^{*} x^{*} y+0.5523^{*} y^{*} y$

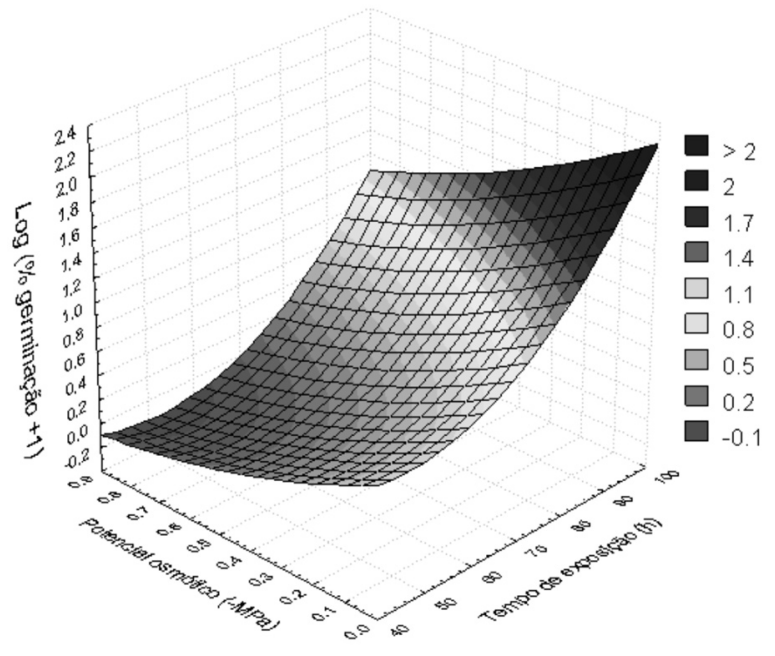

Em Alternaria alternata e Botrytis cinerea o uso de sacarose para alterar o potencial hídrico do substrato proporcionou um aumento no diâmetro médio das colônias dos fungos, ao passo que para Ascochyta paspali o crescimento micelial foi menor a partir do potencial osmótico de -1,2 $\mathrm{MPa}$, sendo inibido a partir de 4,5 MPa (Alam et al., 1996). De acordo com Gao \& Shain (1995), o estímulo ao crescimento fúngico seria proporcionado pela fonte adicional de carbono fornecido por açúcares. A influência positiva da suplementação com sacarose em meio BDA no crescimento radial de $B$. sorokiniana já havia sido demonstrada (Farias et al., 2004) e, no presente estudo, o fungo foi capaz de infectar e colonizar as sementes indiferente do potencial osmótico e tempo exposição avaliados.
FIGURA 1 - Superfície de resposta da germinação de sementes de trigo ao tempo de exposição à cultura de Bipolaris sorokiniana, crescida em meio modificado com adição de sacarose para modificar o potencial osmótico do substrato. Valores percentuais transformados para $\log \mathrm{x}+1$.

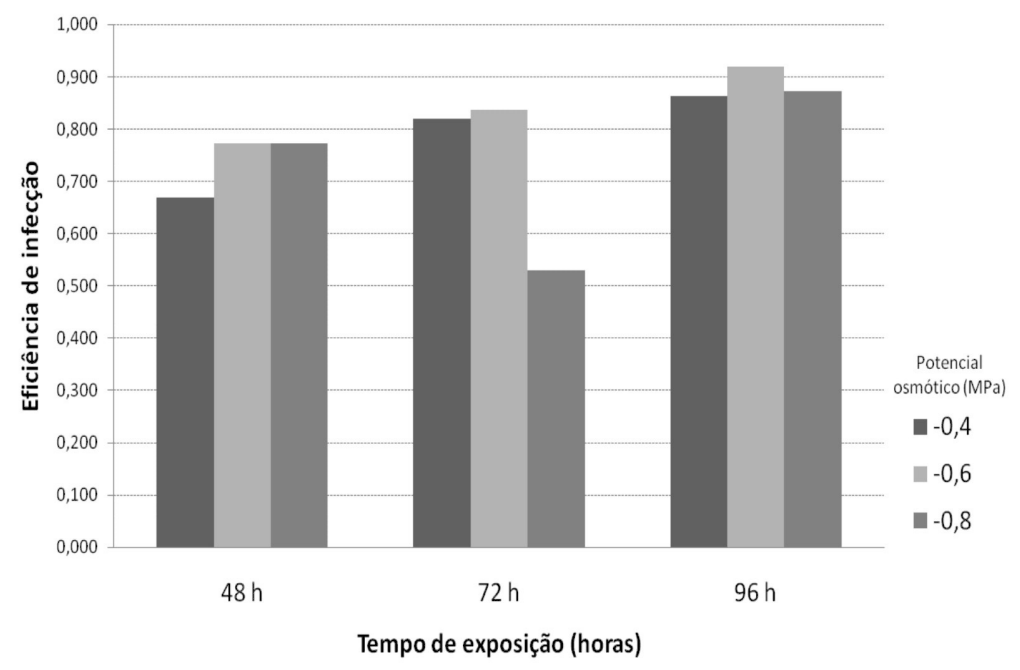

FIGURA 2 - Eficiência média de infecção de sementes de trigo com Bipolaris sorokiniana em função do potencial osmótico do substrato BDA (Batata-DextroseÁgar), ajustado com adição de sacarose, e do tempo de exposição das sementes ao substrato. Efeitos não significativos pelo teste $\mathrm{F}$ da análise de regressão $(\mathrm{P}>0.05)$. 
Embora o aumento do tempo de exposição das sementes ao inóculo aumente a eficiência de infecção, conforme tem sido observado em outros trabalhos, o mesmo pode, por outro lado, causar a diminuição do potencial germinativo da semente em testes de germinação na exposição prolongada em meio com restrição hídrica. $\mathrm{O}$ uso da restrição hídrica em BDA com manitol em níveis $-0,8 \mathrm{MPa}$ foi o tratamento mais eficiente na infecção por Colletotrichum lindemuthianum em sementes de feijão, porém afetou negativamente a capacidade germinativa da sementes, provavelmente em função do longo tempo relativo de exposição (Carvalho, 1999). No presente trabalho, observou-se um significativo decréscimo no percentual de germinação das sementes a partir de $48 \mathrm{~h}$ de tempo de exposição das sementes ao inóculo, não havendo germinação no tempo de 96 horas. Entretanto, a análise de regressão não mostrou efeito significativo segundo o teste $\mathrm{F}$, para os potenciais osmóticos do substrato $(\mathrm{P}>0,05)$ (Figura 3).

Os resultados contrastam com aqueles observados por Araújo et al. (2006), que inocularam sementes de algodão com Colletotrichum gossypii var. cephalosporioides e não verificaram diferenças no percentual de germinação das sementes e de plântulas sobreviventes nos tempos de 36 e 72h, a qual foi reduzida apenas a partir de $108 \mathrm{~h}$ de exposição das sementes. Ainda, Costa et al. (2003) observaram menores percentuais de germinação de sementes de feijão inoculadas com $F$. oxysporum f.sp. phaseoli quando o período de tempo de inoculação foi aumentado de 0 até $144 \mathrm{~h}$. Da mesma forma, em estudos com $X$. campestris pv. phaseoli inoculada em sementes de feijoeiro (Phaseolus vulgaris L.), foi observada uma redução de 10\%

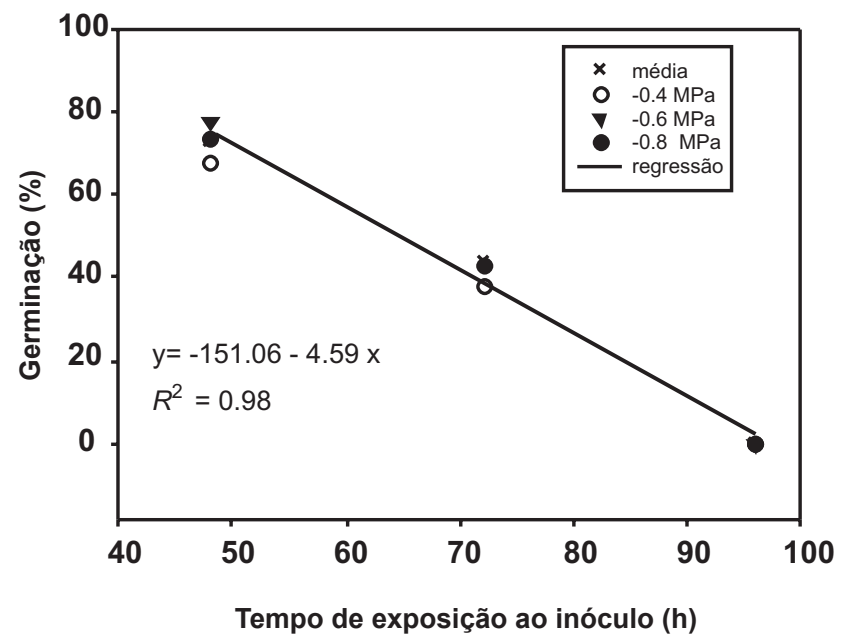

FIGURA 3 - Percentual médio de germinação de sementes de trigo após períodos de tempo de exposição ao inóculo de Bipolaris sorokiniana crescido em meio de cultura modificado osmoticamente com adição de sacarose. Regressão linear significativa usando os valores médios de germinação para os diferentes potenciais osmóticos. na germinação a partir de $36 \mathrm{~h}$ de contato com a bactéria (Valarini \& Menten, 1991). Redução da germinação e conseqüente aumento do percentual de sementes mortas de algodoeiro foram associados à inoculação das mesmas com Fusarium oxysporum f.sp. vasinfectum sob diferentes níveis de restrição hídrica e períodos de exposição de sementes ao patógeno (Araújo et al., 2006; Machado et al., 2007). Esses resultados evidenciaram, portanto, a necessidade de otimizar a técnica de restrição hídrica para cada patossistema. Para o sistema trigo - B. sorokiniana conclui-se que a técnica de restrição hídrica pode ser usada para produzir lotes de sementes infectadas com incidência superior a $75 \%$ não germinadas e com assegurado potencial germinativo, utilizando-se sacarose no substrato para obter potencial osmótico de -0,6 MPa e depositando as sementes por 48 horas sobre a colônia fúngica.

\section{REFERÊNCIAS BIBLIOGRÁFICAS}

Alam S, Joyce D, Wearing A (1996) Effects of equilibrium relative humidity on in vitro growth of Botrytis cinerea and Alternaria alternata. Australian Journal of Experimental Agriculture 36:383388.

Araujo DV, Pozza EA, Machado JC, Zambenedetti EB, Celano FAO, Carvalho EM, Camargo VN (2006) Influência da temperatura e do tempo de inoculação das sementes de algodão na transmissibilidade de Colletotrichum gossypii var. cephalosporioides. Fitopatologia Brasileira 31:35-40.

Bradford KJ (1986) Manipulation of seed water relations via osmotic priming to improve germination under stress conditions. Hortscience 21:1105-1112.

Carvalho JCB (1999) Uso da restrição hídrica na inoculação de Colletotrichum lindemunthianum em sementes de feijoeiro (Phaseolus vulgaris). Dissertação de Mestrado. Universidade Federal de Lavras. Lavras MG.

Costa MLN, Machado JC, Guimarães RM, Guimarães RM, Pozza EA, Oride D (2003) Inoculação de Fusarium oxysporum f. sp. phaseoli em sementes de feijoeiro através de restrição hídrica. Ciência e Agrotecnologia 27:1023-1030.

Coutinho WM, Machado JC, Vieira MGGC, Guimarães RM, Ferreira DF (2001) Uso da restrição hídrica na inibição ou retardamento da germinação de sementes de arroz e feijão submetidas ao teste de sanidade em meio agar-água. Revista Brasileira de Sementes 23:127-135.

Farias CRJ, Del Ponte EM, Correa CL, Pierobom CR (2004) Crescimento radial de Bipolaris sorokiniana em resposta a indução de restrição hídrica por solutos osmóticos em meio agarizado. Revista Brasileira de Agrociência 10:457-460.

Farias CRJ, Del Ponte EM, Dal Magro T (2003) Inibição da germinação de sementes de trigo e milho em testes de sanidade em substrato papel. Revista Brasileira de Agrociência 9:141-144.

Gao S, Shain L (1995) Effect of potential on virulent and hypovirulent strins of the chestnut blight fungus. Canadian Journal of Forest Research 25:1024-1029.

Machado JC, Oliveira JA, Vieira MGGC, Alves MC (2001) Uso 
da restrição hídrica na inoculação de fungos em sementes de milho. Revista Brasileira de Sementes 23:88-94.

Machado JC, Oliveira JA, Vieira MGGC, Alves MC (2004) Uso da restrição hídrica na inoculação de fungos em sementes de algodão. Revista Brasileira de Sementes 26:62-67.

Machado AQ, Machado JC, Carvalho MGGC, Cassetari Neto D, Sousa MV (2007) Potencial do uso da restrição hídrica em testes de sanidade de sementes de algodoeiro. Fitopatologia Brasileira 32:408-414.

Machado JC, Coutinho WM, Guimarães RM, Vieira MGGC, Ferreira DF (2008) Use of osmotic solutes to control seed germination of rice and common bean in seed health blotter tests. Seed Science and Technology 36:66-75.
Neergard P (1979) Seed Pathology. London. Mac Millan Press Ltd.

Reis EM, Forcelini CA (1993) Transmissão de Bipolaris sorokiniana de sementes para órgãos radiculares e aéreos do trigo. Fitopatologia Brasileira 18:76-81.

Toledo J, Reis EM, Forcelini CA (2002) Comparação de métodos para detecção de Bipolaris sorokiniana em sementes de cevada. Fitopatologia Brasileira 27:389-394.

Valarini PJ, Menten JOM (1991) Inoculação artificial de sementes de feijão com Xanthomonas campestris pv. phaseoli e seu efeito sobre a qualidade sanitária e a germinação. Summa Phytopathologica 17:227-231.

TPP 9101 - Recebido 10 Agosto 2009 - Aceito 27 Agosto 2010 Editor de Seção: Jose da Cruz Machado 